\title{
Correction: The psychiatric phenotypes of 1q21 distal deletion and duplication
}

Stefanie C. Linden (1), Cameron J. Watson D, Jacqueline Smith (D, Samuel J. R. A. Chawner (D, Thomas M. Lancaster, Ffion Evans, Nigel Williams, David Skuse (D, F. Lucy Raymond, Jeremy Hall, Michael J. Owen (10, David E. J. Linden, LeeAnne Green-Snyder, Wendy K. Chung, Anne M. Maillard (1), Sébastien Jacquemont (10) and

Marianne B. M. van den Bree (D)

\section{Correction to: Translational Psychiatry https://doi.org/10.1038/s41398-021-01226-9 published online 4 February 2021}

The original version of this article unfortunately contained a mistake in the 'Discussion' section. At the end of the first paragraph, the word 'deletion' should be replaced with 'duplication'. The correct sentence is given below. The authors apologize for the mistake. The original article was revised.
'These findings and the recent report of high prevalence of depression in participants of a population cohort (UK Biobank) with 1q21 duplication underline the association of the 1q21 locus with psychopathology beyond the classical neurodevelopmental disorders'.

Published online: 18 June 2021 